\title{
ANÁLISE ECONÔMICA E AVALIAÇÃO FINANCEIRA DA CULTURA DA MANGA NO CONDOMINIO RURAL TASSO RIBEIRO JEREISSATI NO MUNICIPIO DE MAURITI-CE.
}

\author{
Rosa Dionísio Almeida, Otacio Gomes Pereira Gomes \\ Universidade Federal do Ceará (UFC) \\ <mariarosa_dionisio@hotmail.com>.<otaciopg@gmail.com>
}

10.21439/conexoes.v10i3.849

\begin{abstract}
Resumo. Apesar de sua origem indiana, a mangueira foi introduzida no Brasil pelos portugueses no século XVI, adaptando-se muito bem em várias regiões, sendo muitas vezes confundida como fruteira nativa. O Brasil é um dos maiores produtores de manga do mundo, sendo esta, a segunda fruta mais consumida nos trópicos atrás somente da banana. Rica em sais minerais, vitaminas, açúcares e de sabor muito agradável, a manga é uma das principais frutas consumidas no país. Devido à relevância desta cultura, o presente artigo tem por objetivo analisar economicamente e financeiramente a cultura da manga no Condomínio Rural Tasso Ribeiro Jereissati, localizado nomunicípio de Mauriti, no estado do Ceará. Os dados são provenientes de fontes primárias e secundárias. A metodologia que se utilizou envolveu o cálculo de medidas de resultado econômico, que levou em consideração as medidas residuais e de eficiência, tais como: Taxa de Remuneração do Capital (TRC), Custo Unitário (CU), Ponto de Nivelamento (PNR) e Relação RB/CT, Renda Bruta (RB), Renda Liquida (RL); e o método como avaliação de culturas perenes, empregando a demonstração de fluxo de caixa para definir o valor do pomar no ano 3. Foi concluído que produzir manga é uma atividade economicamente eficiente na qual se obteve o resultado da relação $\mathrm{RB} / \mathrm{CT}=1,9>1$, demonstrado que é uma atividade lucrativapara os produtores desse condomínio, e também estável face às possíveis variabilidades dos custos e receitas, como também mostra um valor do pomar propício à produção.
\end{abstract}

Palavras-chaves: Análise de Investimento. Resultado Econômico. Mauriti.

\begin{abstract}
Despite its Indian origin, the hose was introduced in Brazil by the Portuguese in the sixteenth century, adapting very well in many regions, often mistaken for a native fruit tree. Brazil is one of the largest mango producer in the world, which is the second most consumed fruit in the tropics behind only banana.Rich in minerals, vitamins, sugars and very pleasant flavor, mango is one of the main fruits consumed in the country. Because the relevance of this culture, this article aims to analyze economically and financially the mango crop Farm Tasso RibeiroJereissati Condo located no município of Mauriti in the state of Ceará. The data are derived from primary and secondary sources. The methodology that was used involved the calculation of economic outcome measures, which took into account the waste and efficiency measures, such as: Equity Compensation Fee (TRC),Unit Cost (CU), Leveling Point (PNR) and ratio RB / CT, Gross Income (RB), Net Income (RL); and the method and evaluation of perennial crops, using the cash flow statement to set the value of the orchard in year 3.It was concluded that produce sleeve is a cost-effective activity in which we got the result of the relationship RB / CT $=1.9>1$, demonstrated that it is an activity lucrative to the producers of this condo, and also stable at the possible variability of costs and revenues but also shows a value orchard conducive to production.
\end{abstract}

Keywords: Investment Analysis. Economic Result. Mauriti. 


\section{INTRODUÇÃO}

O Brasil, por sua origem tropical dispõe de fatores propícios ao bom desenvolvimento da fruta, sendo considerado um grande produtor, sua produção é destinada ao abastecimento do mercado interno, como também do mercado externo, produzindo frutas de excelente qualidade (GOMES, 1980).

O cultivo da manga no Brasil historicamente foi feita de maneira extensiva, sendo comum o plantio em áreas extensivas como também nos quintais e fundos de vales das pequenas propriedades. No país, predominam as variedades locais do tipo "Bourbon", "Rosa", "Espada", "Coqueiro", "Ouro", entre outras. Contudo, nos últimos anos, esse quadro vem sendo alterado através da implantação de grandes áreas com novas variedades de manga de comprovada aceitação pelo mercado externo (EMBRAPA, 2010).

O Brasil ocupa a terceira colocação do ranking entre os países produtores de fruta com resultado estipulado em 43,912 milhões de toneladas em 2012. Acima disso estão a China com desempenho gigantesco, de 224,816 milhões de toneladas e a Índia, com 83,032 milhões de toneladas (FAO apud SANTOS, 2013).

A atividade congrega cerca de 5,6 milhões de pessoas, o que corresponde a $34 \%$ da força e trabalho empregado no meio rural, de acordo com o Instituto Brasileiro de Frutas (IBRAF, 2006-2007 apud POLL et al. 2013).

A expansão da mangicultura teve seu pico no Estado de São Paulo, onde foram difundidas as novas variedades para o restante do país, principalmente para os pólos de agricultura irrigada do Nordeste, onde ocorreu à incorporação de plantios tecnificados, principalmente no submédio do Vale do São Francisco com destaque para os estados da Bahia, Pernambuco e outras áreas irrigadas como as dos Vales do Jaguaribe, Açu-Mossoró, Parnaíba e Platô de Neópolis:Ceará, Rio Grande do Norte, Piauí e Sergipe (EMBRAPA, 2010).

No Ceará o município de Mauriti situado na região do Cariri, é um grande produtor de manga por meio da irrigação, principalmente no Condomínio Rural Tasso Ribeiro Jereissati, fonte de pesquisa deste trabalho onde há uma grande incidência desta fruta, constituindo uma fonte de renda para os produtores como é absorvedora de mão-de-obra local.

Para tanto, para que a cultura da mangicultura continue promovendo os resultados satisfatórios é necessário que as propostas discutidas para o desenvolvimento desta atividade, incluam pontos que não vise apenas aspectos agronômicos e econômicos.

Portanto, o presente estudo objetiva analisar economicamente de forma a avaliar a cultura da manga nos
Condomínio Rural Tasso Ribeiro Jereissati no município de Mauriti-Ce.Especificamente, calcularam-se as receitas e custos de implantação e operacionais da atividade comose estimou a produção e a renda líquida obtida.

\section{FUNDAMENTAÇÃO TEÓRICA}

\subsection{Caracterização do cultivo da mangicultura}

Pertencente à família Anacardiácea, a mangueira configura-se como uma das frutas mais populares do mundo. É uma árvore frondosa que pode ser tanto de porte médio como grande, com copa arredondada. A inflorescência 1 gera flores perfeitas, tem como polinizador a mosca doméstica, o fruto é variável no tamanho, peso, forma e cor, a polpa é amarela (de vários tons), de sabor variado dependendo da variedade. Adaptamse melhor em áreas onde as estações secas e chuvosas são bem definidas, vegeta e frutifica tanto em solos arenosos como em solos argilosos ligeiramente ácidos ou alcalinos (FERREIRA; CASTRO, 2001).

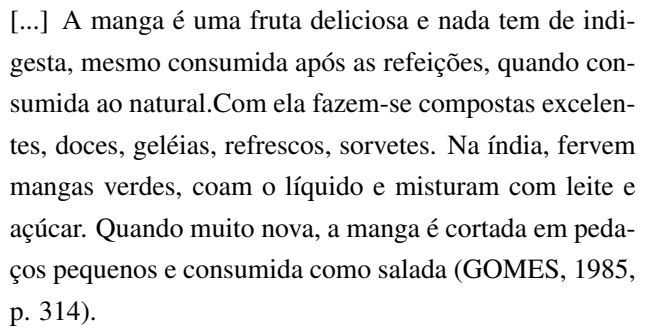

A Organização das Nações Unidas para Alimentação e Agricultura (FAO) estima que a colheita de manga será de cerca de 28,8 milhões de toneladas em 2014, ou seja, $35 \%$ da produção mundial de frutas tropicais (FAO apud TODA FRUTA.COM, 2014). No Brasil é muito grande a variedade de mangas que podemos encontrar entre as quais; Espada, Rosa, Haden, keitt, Kent, TomyAtkions, Palmer, Van Dyke entre outras. As variedades mais indicadas são as que apresentam alta produtividade, coloração atraente do fruto, preferencialmente avermelhado, polpa doce, pouca ou nenhuma fibra, além da resistência ao manuseio e ao transporte para mercados distantes.

Atualmente, a Tommy Atkins é a variedade mais produzida e a que possui a maior participação no volume comercializado no mundo, devido principalmente a sua coloração intensa, produções elevadas e resistência ao transporte a longas distâncias (EMBRAPA. 2014).

\footnotetext{
${ }^{1}$ Estrutura vegetal formada pelo agrupamento de flores em um mesmo pendúlo. Os principais tipos de inflorescência são: racemo, espiga, umbela, cimeira, capitulo e carimbo. Ou o conjunto dessas flores.
} 
Tabela 1: Produção brasileira de mangicultura por regiões em 2013.

\begin{tabular}{c|c|c|c|c}
\hline $\begin{array}{c}\text { Região } \\
\text { fisiográfica }\end{array}$ & $\begin{array}{c}\text { Área colhida } \\
\text { (ha) }\end{array}$ & $\begin{array}{c}\text { Quantidade } \\
\text { produzida (t) }\end{array}$ & $\begin{array}{c}\text { Valor da } \\
\text { produção (Mil } \\
\text { Reais) }\end{array}$ & $\begin{array}{c}\text { Participação na } \\
\text { produção (\%) }\end{array}$ \\
\hline Norte & 197 & 1.351 & 803 & $0,03 \%$ \\
Nordeste & 48.625 & 784.281 & 648.407 & $8,03 \%$ \\
Sudeste & 20.490 & 363.801 & 242.950 & $1,20 \%$ \\
Sul & 730 & 9.292 & 10.672 & $0,23 \%$ \\
Centro-Oeste & 330 & 4.275 & 4.095 & $0,52 \%$ \\
\hline Brasil & $\mathbf{7 0 . 3 7 2}$ & $\mathbf{1 . 1 6 3 . 0 0 0}$ & $\mathbf{9 0 6 . 9 2 7}$ & $\mathbf{1 0 0 , 0 0 \%}$ \\
\hline
\end{tabular}

Fonte: IBGE- Produção Agrícola Municipal 2013. Consultado em 06/08/2015.

Tabela 2: Produção brasileira de mangicultura por estados do Nordeste em 2013.

\begin{tabular}{|c|c|c|c|}
\hline Estados & $\begin{array}{l}\text { Área Colhida } \\
\text { (ha) }\end{array}$ & $\begin{array}{c}\text { Produção } \\
\text { (t) }\end{array}$ & $\begin{array}{l}\text { Valor da produção } \\
\text { (Mil Reais) }\end{array}$ \\
\hline Bahia & 25.412 & 437.919 & 242.280 \\
\hline Pernambuco & 10.372 & 214.117 & 326.375 \\
\hline Ceará & 5.576 & 46.632 & 23.413 \\
\hline Rio Grande do Norte & 2.691 & 38.115 & 24.092 \\
\hline Sergipe & 846 & 19.198 & 15.894 \\
\hline Paraiba & 1.527 & 11.039 & 8.087 \\
\hline Piaui & 856 & 7.847 & 3.211 \\
\hline Alagoas & 799 & 6.378 & 1.993 \\
\hline Maranhão & 552 & 3.036 & 3.062 \\
\hline BRASIL & 73.310 & 1.163 .000 & 906.927 \\
\hline
\end{tabular}

Fonte: IBGE- Produção Agrícola Municipal 2013. Consultado em 06/08/2015.

As regiões Nordeste e Sudeste se destacam na produção nacional de manga, foram responsáveis no ano 2013, respectivamente, por 782.281 e 363.801 toneladas da produção total de manga, enquanto que a região Sul produziu 9.292 toneladas, na região Norte produziu-se apenas $1.351 \mathrm{t}$ e o Centro-Oeste um pouco mais que a anterior sendo 4.275 toneladas, conforme a Tabela1 1

De acordo com a Tabela 2 dentre todos os estados nordestinos, os estados da Bahia e Pernambuco se destacam na produção de manga em relação aos aspectos como área colhida, produção e valor da produção, com respectivamente 25.412 e 10.372 hectares de área colhida, 437.919 e 214.117 toneladas de fruta produzida, constituindo um valor de 242.280 e 326.375 mil reais em 2013. Nos estados da Bahia e Pernambuco, os municípios de Juazeiro (BA) e Petrolina (PE) caracterizam-se como referência na produção de manga (IBGE, 2013).

A despeito da região de Petrolina /Juazeiro em produzir manga durante o ano todo, sua produção está concentrada nos meses de julho até dezembro, as exportações nordestinas sucedem em maior expressão nos meses de agosto a dezembro, destinando-se aos mercados americano e para a Europa, onde a fruta é recebida no primeiro semestre, porque o volume de produção é menor (CINTRA, 2002).

\section{METODOLOGIA}

\section{1 Área de estudo}

O município de Mauriti está localizado ao Sul do Estado do Ceará, distante $406 \mathrm{~km}$ da capital cearense em linha reta. De acordo com Instituto de Geografia e Estatística (IBGE, 2010), sua população é de 44.240 habitantes. Detêm uma área territorial de $1111,86 \mathrm{~km}^{2}$, limita-se ao norte com Estado da Paraíba e o município do Barro; ao sul faz limite com o município de Brejo Santo, e os Estados da Paraíba e Pernambuco; ao leste com Estado da Paraíba, já a oeste com os municípios de Brejo Santo e Milagres. Possui um clima tropical quente semiárido, a pluviosidade é de $872,3 \mathrm{~mm}$, os meses mais chuvosos são fevereiro á abril. A temperatura média é de $24^{\circ} \mathrm{C}$ a $26^{\circ} \mathrm{C}$ (IPCE, 2013).

\subsection{Fonte dos dados}

Os dados primários quantitativos e qualitativos foram coletados junto aos produtores do Condomínio Rural Tasso Ribeiro Jereissati do município de Mauriti- CE. A forma de entrevista foi semiestruturada, que de acordo com Triviños (1987), tem como particularidade, questionamentos básicos que são sustentados em hipóteses e teorias que estão relacionados ao tema da pesquisa.

O período de coleta dos dados foi realizado no mês de julho de 2014. Portanto, os preços dos insumos, serviços, fatores de produção e preço do produto correspondem a este período. As seguintes técnicas de pesquisa para a coleta de dados foram usadas: pesquisa bibliográfica, pesquisa documental e pesquisa empírica.

\subsection{Método de Análise}

\subsubsection{Análise Econômica da Produção de Manga}

Para se avaliar a eficiência econômica é importante entender os conceitos de receitas e custos da produção, para depois se chegar aos indicadores propostos no mencionado trabalho e conseguir os resultados esperados.

O conceito de custo é definido por "o valor de bens e serviços consumidos na produção de outros bens ou serviços"(MATTOS, 1998). Portanto, está relacionado com todos os bens e serviços que uma definida atividade produtiva utiliza num certo período de tempo, definindo-se como as despesas (saídas) da atividade implantada.

Já receitas, conforme Silva (1999) tomando U como utilidade (serviço ou bem), no qual preço de venda por unidade signifique um preço fixo $\mathrm{P} 0$, para quantias entre q1 e q2 unidades. A função dada por RT=P0.q, com 
$\mathrm{q} 1 \leq \mathrm{q} \leq \mathrm{q} 2$, é designada função receita total ou simplesmente receita total (valor total recebido por um número de produtos vendidos a um certo preço fixo).

O método utilizado nesse trabalho para a análise econômica levou em consideração que todos os fatores utilizados devem ser remunerados para produzir determinado bem, compreendendo-se entre eles, empresário, terra e capital. É importante ainda observar que o conceito clássico de custo se fundamenta em dois aspectos basais: a) no princípio do custo de oportunidade ou custo alternativo, no qual o custo dos recursos para a empresa é igual ao seu valor no mais perfeito uso alternativo; e, b) nos custos implícito e explícito; o primeiro é acatado como os encargos devidos a fatores que pertencem à firma, o segundo, é definido como a despesa em dinheiro comumente realizado pela firma, referente aos gastos com serviços alugados ou comprados (MATSUNAGA et al.,1976).

Como já recomendado, o custo de produção considera todos os itens que ficassem indireta ou diretamente ligados ao processo de produção, aceitando-se critérios subjetivos para recompensar determinados fatores.

De forma geral, o custo de produção expõe os seguintes componentes:

a) Despesas indiretas (CV), que são levados em consideração os gastos em dinheiro com fertilizantes, aluguel de equipamentos, trabalhadores, transportes, defensivos, etc.

b) Despesas diretas (CF): fazem parte taxas e impostos, gastos gerais e diversos, entre estes estão listados luz e força, água, material de escritório, conservação de benfeitorias, instalações, máquinas e equipamentos e depreciações. Para o cálculo da depreciação, utilizouse a seguinte fórmula de acordo com Hofmann et al. (1987):

$$
D=\frac{V_{i}+V_{f}}{n}
$$

Onde:

$V_{i}=$ Valor inicial do bem de capital;

$V_{f}=$ Valor final do bem de capital;

$n=$ Tempo de vida útil do bem de capital;

c) Remuneração dos demais componentes: terra e capital

Segundo Hofmann et al. (1987), utilizando-se os indicadores de resultados econômicos é possível avaliar o rendimento de uma atividade, ou seja, algumas medidas de lucratividade e eficiência econômica de uso dos fatores de produção.

Os indicadores de resultados econômicos utilizados são:

- Renda Bruta (RB);
- Renda Líquida (RL);

- Taxa de Remuneração do Capital (TRC);

- Custo Unitário (CU)

- Ponto de Nivelamento de Rendimento (PNR);

- Relação Renda Bruta/Custo Total (RB/CT).

Como forma de obter os indicadores acima referidose para que haja melhor compreensão do tema, é necessário definir esses e outros indicadores de resultados econômicos, definidos pelo autor mencionado,descritos a seguir:

(a) Renda Bruta (RB) - abrange a soma dos valores encontrados como resultado da atividade, em formas monetárias por forma matriz, tais como:

- renda bruta da venda da manga;

- renda bruta do autoconsumo;

(b) Renda Líquida (RL) - este indicador é tido como a diferença entre a renda bruta (RB) e as despesas (D).

$$
R L=R B-D
$$

(c) Remuneração do Capital (RC) - é dado pelo valor do capital que está empatado na propriedade multiplicado pela taxa de juros do FNE (6,75\% a.a.).

(d) Renda da Terra (RT) - é dada pelo produto do valor da terra empregada na produção pela taxa do FNE $(6,75 \%$ a.a. $)$.

(e) Taxa de Remuneração do Capital (TRC) - é a remuneração pelo uso do capital, do que sobra depois de descontada todas as despesas de operação inclusive a remuneração do empresário.Assim, conforme Hofmann et al. (1987):

$$
T R C=\frac{R C}{C M E} x 100
$$

Em que:

$$
C M E=\frac{I_{i}+I_{f}}{2}
$$

CME = capital médio empatado;

$\mathrm{Ii}=$ valor total do inventário no início do ano;

If = valor total do inventário no fim do ano.

(f) Custo Unitário (CU) - indica quanto o produtor gasta em unidades monetárias para produzir um kg de manga. Para chegar a este valor, divide-se o custo total (CT) pelo volume físico de produção (VFP). Levando em consideração a conceituação teórica do custo total de produção, no qual o CT é formado por todos os gastos da atividade, inclusive a remuneração do capital e da terra. Hofmann et al. (1987):

$$
C U T=\frac{C T}{V F P}
$$

(g) Ponto de Nivelamento de Rendimento (PNR) o Ponto de Nivelamento de Rendimento mostra como 
se comportam os custos e as receitas e, também, de seus componentes, em certo período de tempo. Os valores foram obtidos dividindo-se o Custo Total (CT) pelo preço médio do kg da manga. Hofmann et al. (1987):

$$
P N R=\frac{C T}{\text { preço médio venda }}
$$

(h) Relação RB/CT - A relação Renda Bruta/Custo Total mostra o comportamento das receitas relacionadas aos custos totais da atividade, acordados com as situações a seguir: $R B / C T=1$, situação regular, receitas iguais aos custos totais; $R B / C T>1$, situação boa, onde as receitas superam os custos totais; $R B / C T<1$, situação ruim, pois os custos totais superam as receitas, ou seja, é gerado prejuízo.

\subsubsection{Avaliação da Cultura da Manga}

De forma conceitual, o valor do pomar (manga) é estimado baseado nos gastos da fase de formação e o valor presente da renda líquida calculado para cada um dos anos futuro do pomar. Desta forma para qualquer ano "n" na fase de produção, o valor do pomar é calculado de acordo com HOFMANN et al. (1987):

$$
V P_{n}=V F F_{n}+V P R L F *(1-r)
$$

Onde:

$\mathrm{VP}_{n}=$ Valor do pomar no ano "n";

$\mathrm{VFF}_{n}=$ Valor futuro dos gastos de formação da cultura;

VPRLF $=$ Valor Presente das Receitas Líquidas Futuras (anos posteriores ao ano " $n$ ");

$\mathrm{r}=$ Coeficiente de risco que está ligado à queda no fluxo de receitas líquida por conta de imprevistos que acabem resultando em queda no preço do produto e/ou na produtividade almejada e/ou nos valores dos insumos, comumente atribui-se uma taxa de $10 \%$ ao risco.

Em conformidade com o autor citado anteriormente, para calcular o Valor Futuro dos Gastos de Formação (VFF) no ano "n" tem-se:

$$
V F F_{n}=\sum_{i=0}^{n} C F^{i} *(1+r)^{n-1}
$$

Em que:

$\mathrm{CF}=$ Custo de Formação da cultura.

$\mathrm{i}=$ anos da formação;

$\mathrm{n}=$ Quantidade de anos da formação da cultura;

$\mathrm{r}=$ Taxa de juros reais;

Para o cálculo do Valor Presente das Receitas Líquidas Futuras (VPRLF) no ano "3” (período em que a cultura é estabilizada), como sugerido, tem-se:
Tabela 3: Cronograma de valor do capital empatado por hectare, para os produtores de manga do Condomínio Rural Tasso Ribeiro Jereissati, município de Mauriti, Estado do Ceará, 2014.

\begin{tabular}{l|c|c|c|c}
\hline Inversões (ano zero) & $\begin{array}{c}\text { Vida Útil } \\
\text { restante } \\
\text { (anos) }\end{array}$ & $\begin{array}{c}\text { Valor Inicial } \\
\text { (RS) }\end{array}$ & $\begin{array}{c}\text { Valor Final } \\
\text { (RS) }\end{array}$ & $\begin{array}{c}\text { Depreciação } \\
\text { (RS) }\end{array}$ \\
\hline - Terreno & - & $8.000,00$ & $8.000,00$ & - \\
\hline - Galpão & 20 & $6.000,00$ & $5.700,00$ & 300 \\
\hline - Máquinas e Equipamentos & 8 & $5.000,00$ & $4.375,00$ & 625 \\
\hline - Canos & 10 & 180 & 162 & 18 \\
\hline - Gotejadores e outros & 2 & 860 & 430 & 430 \\
\hline Total & & $\mathbf{2 0 . 0 4 0 , 0 0}$ & $\mathbf{1 8 . 6 6 7 , 0 0}$ & $\mathbf{1 . 3 7 3 , 0 0}$ \\
\hline
\end{tabular}

Fonte: Dados da pesquisa (2014)

$$
V P R L F_{m}=\sum_{j=4}^{k} \frac{R L_{j}}{(1+r)^{k}}
$$

Onde:

RL = Receita Líquida;

$\mathrm{m}=$ ano 3 (período de estabilização da cultura);

$\mathrm{k}=$ Quantidade de anos restante do fluxo da cultura; $\mathrm{j}=$ Anos seguintes ao do valor em análise $(\mathrm{m}=3)$.

\section{RESULTADOS E DISCUSSÃO}

Primeiramente foi feito a análise das medidas de resultado econômico e depois se procedeu a análise da avaliação da cultura da manga, especificamente, o valor do pomar para o ano 3 .

\subsection{Levantamento dos Investimentos e Custos Operacionais}

A Tabela 3 mostra os valores dos equipamentos para se produzir um hectare de manga, supondo o sistema de irrigação incluindo apenas canos, gotejadores e "outros" no qual está incluso a instalação, a terra para o cultivo da manga, galpão, máquinas e equipamentos, com as respectivas quantidades e a vida útil de cada fator, com um valor inicial total de $\mathrm{R} \$ 20.040,00$; valor final de $\mathrm{R} \$ 18.667,00$, com custo de depreciação total igual a $\mathrm{R} \$ 1.373,00$

A Tabel/ 4 apresenta os custos operacionais para produção da manga, estes são compostos pelos serviços de roçagem e aração, gradagem e calagem, covas (sulcamento), mão de obra, roçadeira, pulverização, colheita, além de insumos e fertilizantes. Assim sendo, as despesas indiretas ou os custos variáveis estimados da produção de manga irrigada do condomínio rural Tasso RibeiroJereissati são de $\mathrm{R} \$ 6.720,00$ a partir do $3^{\circ}$ ano em diante.

$\mathrm{Na}$ Tabela 5 podemos analisar a renda bruta que corresponde a toda a receita do negócio, na qual podemos 
ANÁLISE ECONÔMICA E AVALIAÇÃO FINANCEIRA DA CULTURA DA MANGA NO CONDOMINIO RURAL TASSO RIBEIRO JEREISSATI NO MUNICIPIO DE MAURITI-CE.

Tabela 4: Custos variáveis ou despesas indiretas por hectare dos produtores de manga do Condomínio Rural Tasso Ribeiro Jereissati, município de Mauriti, Estado do Ceará, 2014.

\begin{tabular}{|c|c|c|c|c|c|c|c|c|}
\hline \multirow[t]{2}{*}{ Discriminação } & \multirow[t]{2}{*}{\begin{tabular}{|l} 
Unid. \\
\end{tabular}} & \multirow[t]{2}{*}{$\begin{array}{c}\text { Preço } \\
\text { Unitário }\end{array}$} & \multicolumn{2}{|c|}{ Ano Zero } & \multicolumn{2}{|c|}{$1^{\circ} \mathrm{Ano}$ ao $2^{\circ} \mathrm{Ano}$} & \multicolumn{2}{|c|}{$\begin{array}{l}3^{\circ} \text { Ano em } \\
\text { diante. }\end{array}$} \\
\hline & & & Quant & Valor & \begin{tabular}{|l|} 
Quant \\
\end{tabular} & Valor & \begin{tabular}{|l|} 
Quant \\
\end{tabular} & Valor \\
\hline $\begin{array}{l}\text { 1. Preparo Solo e } \\
\text { Plantio }\end{array}$ & & & & 2400 & & & & \\
\hline - Roçagem $\mathrm{e}$ aração & trih & 0,60 & 5 & 1500 & - & - & - & - \\
\hline - Gradagem e Calagem & trh & 0,60 & 5 & 600 & - & - & - & - \\
\hline - Covas (sulcamento) & th/h & 0,60 & 5 & 300 & - & & - & - \\
\hline 2. Insumos & & & & 2994 & & 1100 & & 1100 \\
\hline Mudas & Um & 4,00 & 310 & 1240 & - & - & - & - \\
\hline Agua & $\mathrm{m}^{3}$ & 250 & 1 & 250 & 1 & 250 & 1 & 250 \\
\hline Energia & Kwh & 250 & - & - & 1 & 250 & 1 & 250 \\
\hline \multicolumn{9}{|l|}{ Adubos minerais: } \\
\hline - Sulfato de Potássio & $\mathrm{Kg}$ & 1,36 & 50 & 68 & - & - & - & - \\
\hline Fosforo & $\mathrm{Kg}$ & 1,36 & 50 & 68 & - & - & - & - \\
\hline Nitrogênio & $\mathrm{Kg}$ & 1,36 & 50 & 68 & - & - & - & - \\
\hline Magnésio & $\mathrm{Kg}$ & 28 & 25 & 700 & - & - & - & - \\
\hline Calcário & t. & 250 & 1 & & - & - & - & - \\
\hline Gesso & t. & 300 & 1 & & - & - & - & - \\
\hline Defensivos & L & 150 & 4 & 600 & 4 & 600 & 4 & 600 \\
\hline $\begin{array}{l}\text { 3. Tratos culturais } \mathrm{e} \\
\text { fitossanitários }\end{array}$ & & & & 3300 & & 3300 & & 3300 \\
\hline Măo de obra & h/d & 180 & 10 & 1800 & 50 & 1800 & 50 & 1800 \\
\hline Roçadeira & trh & 60 & 5 & 300 & 5 & 300 & 15 & 900 \\
\hline Pulverização & tr/h & 120 & 10 & 1200 & 10 & 1200 & 10 & 1200 \\
\hline 4. Colheita & $\mathrm{h} / \mathrm{d}$ & $\frac{120}{60}$ &  & - & - & - & 20 & 1200 \\
\hline
\end{tabular}

Fonte: Dados da pesquisa (2014).

verificar um valor de $\mathrm{R} \$ 25.000,00$. No que se referem aos custos fixos estes foram de $\mathrm{R} \$ 3.157,675$ que são compostos pela depreciação dos bens de capital, manutenção e gastos com água. Os custos variáveis ou as despesas indiretas, onde estão calculadas as despesas operacionais da atividade somaram R $\$ 900,00$.

A renda líquida que é o lucro da propriedade foi de $\mathrm{R} \$$ 18.000. A taxa de remuneração do capital foi $6,5 \%$ que representa a remuneração pela utilização do capital, subtraídas todas as despesas de operação. O custo unitário, ou seja, por $\mathrm{kg}$ do produto foi $\mathrm{R} \$ 0,16$ que representa o preço mínimo que o produtor gasta em unidades monetárias para produzir um kg de manga, valores estes variáveis se levarmos em consideração a época do ano, como fatores que interferem no rendimento produção; clima, investimentos, demanda, entre outros.

A variedade de manga que predomina no Condomínio agrícola é a Tommy Atkins, a mais resistente ao transporte de longas distâncias como é mais comercializada.

A produção de manga no Condomínio Rural Tasso Ribeiro Jereissati destina-se ao mercado local, estados da Paraíba e Pernambuco, e as Centrais de Abastecimento do Ceará (CEASA).

Levando em conta também o ponto de nivelamento do rendimento (PNR) que representa o mínimo que deve ser produzido para que as receitas igualem aos custos, identificou-se valor igual a $3.381,4 \mathrm{~kg}$ e a relação $R B / C T=1,9>1$, ou seja, mostra que a atividade da mangicultura é lucrativa, o que beneficia os
Tabela 5: Indicadores econômicos da produção de manga irrigada no perímetro irrigado Senador Tasso Jereissati, município de Mauriti, Estado do Ceará, 2014.

\begin{tabular}{l|c}
\hline \multicolumn{1}{c|}{ ITENS } & TOTAL \\
\hline 1. RENDA BRUTA & 25.000 \\
\hline 2. DESPESAS DIRETAS (CF) & 3157.675 \\
\hline 2.1. Depreciação anual & 600 \\
\hline 2.2. Juros sobre o capital & 1.000 \\
\hline 2.3. Juros sobre a terra & 540 \\
\hline 2.4. Manutenção: 5\% sobre o capital & 767.675 \\
\hline 2.5. Água & 250 \\
\hline 2.6. Encargos Sociais & 737,28 \\
\hline 3. DESPESAS INDIRETAS & 900 \\
\hline 4. TOTAL DESPESAS (CT) & 4057.675 \\
\hline 5. RENDA LíQUIDA (lucro) & 18000 \\
\hline 6. TAXA DE REM. CAPITAL & 6,5 \\
\hline 7. CUSTO UNITÁRIO/Kg & 0,16 \\
\hline 8. PNR (Ponto Nivelamento Rendimento) & 3381,4 \\
\hline 9. RB/CT & 1,9 \\
\hline
\end{tabular}

Fonte: Dados da pesquisa (2014).

Tabela 6: Fluxo de caixa dos custos e receitas da produção de manga no Condomínio Rural Tasso Jereissati, município de Mauriti, Estado do Ceará, 2014.



Fonte: Dados da pesquisa (2014)

produtores possibilitando um rendimento necessário ao atendimento das necessidades básicas e familiares.

\subsection{Avaliação da Cultura da Manga}

De acordo com a Tabela6 acima, para os resultados da avaliação da cultura de manga no Condomínio Rural Tasso Ribeiro Jereissati, considerou-se o fluxo de caixa para um horizonte de 10 anos, instrumento necessário para o cálculo do valor do pomar no ano 3 (período de estabilização da cultura), em que mostra o total de entradas (receitas) e saídas (custos mais investimentos), os gastos futuros da formação (VFF) que somam um total de R\$ 73.018,20 e o Valor Presente das Receitas Líquidas Futuras (VPRLF) para o ano 3, num total de $\mathrm{R} \$ 61.429,80$, assim o valor do pomar no ano 3 foi de 
ANÁLISE ECONÔMICA E AVALIAÇÃO FINANCEIRA DA CULTURA DA MANGA NO CONDOMINIO RURAL TASSO RIBEIRO JEREISSATI NO MUNICIPIO DE MAURITI-CE.

$\mathrm{R} \$ 128.305,00$

\section{CONCLUSÕES}

No município de Mauriti-Ce a produção da mangicultura tem grande impacto na melhoria da qualidade de vida dos produtores do Condomínio Rural Tasso Ribeiro Jereissati, contribuindo para o aumento da renda destes,possibilitando o atendimento básico das necessidades familiares, a renda líquida obtida com a atividade chega $\mathrm{R} \$ 18.000,00$ podendo atingir valores maiores, em anos de melhores condições.

O referido trabalho foi discutido em perspectiva da análise econômica da produção de manga irrigada, como também para avaliação do pomar em um determinado período produtivo. Como mostram os indicadores econômicos, o projeto mostrou viabilidade, expondo resultados positivos para os indicadores que foram calculados, isto é, segundo os custos e receitas apresentados no estudo, as receitas mostraram-se maiores que os custos.

A relação $R B / C T=1,9>1$ mostra que a atividade da mangicultura é lucrativa, contribuindo para viabilidadedo projeto, promovendo também garantia de renda e geração de emprego aos produtoresque vivem desta prática agrícola.

\section{REFERÊNCIAS}

CINTRA, R. F.; BOTEON, M. Avaliação do desempenho regional dos principais pólos produtores de manga no brasil. In: Anais do Congresso Brasileiro de Economia e Sociologia Rural. São Paulo: [s.n.], 2002. Disponível em: <http://cepea.esalq.usp.br> Acesso em: 15 Set. 2014

EMBRAPA. Empresa Brasileira de Pesquisa Agropecuária. 2010. Disponível em: <http: //www.sct.embrapa.br/novosite/embrapa/acs/emb30an/ areasdegr.html> Acesso em: 10 jan. 2014.

Empresa Brasileira de Pesquisa Agropecuária

- Sistemas de Produção. 2014. Disponível em: <http://sistemasdeproducao.cnptia.embrapa.br.html> Acesso em: 25 Set. 2014

FAO. FOOD AND AGRICULTURE ORGANIZATION. Mangifera Indica, L. Austrália. 2014. Disponível em: <http://www.fao.com> Acesso em: 20 Set. 2014.

FERREIRA, C. C.; CASTRO, E. B. Curso de Produção da Manga. Limoeiro do Norte- CE: Secretaria de Agricultura Irrigada, 2001. 30 p.
GOMES, R. P. Fruticultura Brasileira. 6. ed. São Paulo: Nobel, 1980.

HOFFMAN, R.; ENGLER, J. D. C.; SERRANO, O.; THAME, A. C. M.; NEVES, E. M. Administração da empresa agrícola. São Paulo: Pioneira, 1987. 320 p.

IBGE. Instituto brasileiro de geografia e estatística. dados da produção e consumo de manga no brasil. 2013. Disponível em: <http://www.sidra.ibge.gov.br> Acesso em: 10 Agosto. 2015.

IBRAF. Comparativo das exportações brasileiras de frutas frescas. 2006-2007. Disponível em: <www.ibraf.org.br/EstatísticaseRevista>

IPECE. Perfil Básico Municipal. Fortaleza: Instituto de Pesquisa Econômico do Ceará, 2013. Disponível em: $<$ http//www.ipece.ce.gov.br> Acesso em: 18 Ago. 2014.

LAROUSSE. Dicionário enciclopédico ilustrativo Lorousse. São Paulo: Larousse do Brasil, 2007.

MATSUGA, M.; BEMELMANS, P. F.; TOLEDO, P. E. N.; DULLEY, R. D.; OKAWA, H.; PEDROSO, I. A. Metodologia de custo de produção utilizada pelo IEA. Agricultura em São Paulo, São Paulo, v. 23, n. 1, p. 123-139, 1976.

MATTOS, J. C.; TOLEDO, J. C. Custos da qualidade: diagnóstico nas empresas com certificação iso 9000. Revista Gestão \& Produção, São Carlos, v. 5, n. 3, 1998.

POLL, H.; KIST, B. B.; SANTOS, C. E.; REETZ, E. R.; CARVALHO, C.; SILVEIRA, D. N. Anuário brasileiro da fruticultura 2013. Editora Gazeta Santa Cruz, Santa Cruz do Sul, p. 136, 2013.

SILVA, J. P. Análise financeira das empresas. 4. ed. São Paulo: Atlas, 1999.

TODAFRUTA. TODAFRUTA.COM. 2014. Disponível em: <http//www.todafruta.com.br $>$ Acesso em: 15 Set. 2014.

TRIVIÑOS, A. N. S. Introdução à pesquisa em ciências sociais: a pesquisa qualitativa em educação. São Paulo: Atlas, 1987. 